\title{
Circuit
}

Musiques contemporaines

\section{Montréal/Nouvelles Musiques}

Compte rendu

\section{Festival Report}

\section{Nicolas Gilbert et Maxime McKinley}

Volume 14, numéro 2, 2004

Montréal/Nouvelles Musiques

URI : https://id.erudit.org/iderudit/902311ar

DOI : https://doi.org/10.7202/902311ar

Aller au sommaire du numéro

Éditeur(s)

Les Presses de l'Université de Montréal

ISSN

1183-1693 (imprimé)

1488-9692 (numérique)

Découvrir la revue

Citer ce document

Gilbert, N. \& McKinley, M. (2004). Montréal/Nouvelles Musiques : compte rendu. Circuit, 14(2), 35-48. https://doi.org/10.7202/902311ar

\section{Résumé de l'article}

Les deux auteurs présentent leurs points de vue personnels sur la première édition du festival international Montréal/Nouvelles Musiques qui s'est déroulée au mois de mars 2003. Les événements et les oeuvres qui ont été, à leur avis, les plus marquants sont revisités parfois avec sérieux, parfois avec une certaine légèreté, mais toujours en tentant de rester dans l'esprit du festival et des oeuvres qu'il a mises de l'avant. d'utilisation que vous pouvez consulter en ligne.

https://apropos.erudit.org/fr/usagers/politique-dutilisation/ 


\title{
Montréal/Nouvelles Musiques Compte rendu
}

\author{
Nicolas Gilbert et Maxime McKinley
}

Du 2 au 11 mars 2003 avait lieu à Montréal l'événement Montréal/Nouvelles Musiques (MNM), présenté par la Société de musique contemporaine du Québec en partenariat avec la Faculté de musique de I'Université McGill, I'Orchestre symphonique de Montréal, la Chaîne culturelle de Radio-Canada et CBC Radio Two. Cette toute première édition du festival international qui deviendra bisannuel mettait à l'honneur les Pays-Bas. Durant les 10 jours d'activités, le public a eu droit à 19 concerts, huit conférences et cinq classes de maîtres, le tout faisant intervenir plus de 300 musiciens et une cinquantaine de compositeurs.

Nous présentons ici nos impressions sur certains des événements les plus marquants du festival.

\section{Démons et merveilles - Ensemble de la SMCQ}

Si MNM se veut une sorte de "choc électrique" visant à faire "exploser" la nouvelle musique canadienne sur la scène internationale, le Ballet mécanique de George Antheil, œuvre qui ouvrait le festival, peut être considéré comme le symbole de ce choc. Dès le premier instant, cette œuvre s'impose comme une immense déflagration musicale. Liée de près à l'esprit du futurisme, tel que défini d'abord par I'Italien Filippo Marinetti puis par l'écrivain russe Vladimir Maïakovski, cette musique nous entraîne dans un monde d'excès. Les thèmes chers à Marinetti, la machine, la vitesse, la ville, des thèmes touchant tous d'assez près la révolution industrielle, sont omniprésents dans le Ballet mécanique. Musicalement, c'est l'aspect rythmique qui domine, menant la pièce, tel un train roulant à toute vitesse, à travers l'espace de notre perception. La présence des sirènes, des cloches et des moteurs 
confère à l'œuvre son atmosphère d'urgence, presque d'insurrection. Ce qui est urgent, on ne peut en douter, c'est le changement. C'est justement son enthousiasme pour le changement, pour l'arrivée d'un monde nouveau et meilleur où l'homme et la machine seront réunis pour le bien de l'humanité, qu'Antheil veut nous communiquer. Le Ballet mécanique, cette ode au $\mathrm{XX}^{\mathrm{e}}$ siècle, porte donc fièrement les couleurs des années folles, années de tous les scandales.

Le programme de ce concert d'ouverture n'a pas été conçu au hasard : esthétiquement, les deux pièces qui le composent sont situées aux antipodes l'une de l'autre. Les excès de vitesse et d'intensité de l'œuvre de George Antheil font place, dans Pohjatuuli de Michel Longtin, à la narration, à la douceur, aux subtilités d'un univers poétique tout en nuances. Le Ballet mécanique portait sur les machines et la ville, tandis que l'œuvre de Longtin se rapporte à la nature et à ses merveilles. L'œuvre répond au modèle de la "narration onirique", c'est-à-dire qu'elle présente certains personnages musicaux (ici, Sibelius, les paysages du nord et la conscience de l'artiste, telle qu'exprimée par la partie de clarinette solo, l'instrument-pensant de l'œuvrel, qui interagissent et suivent un parcours musical non pas rectiligne, mais sinueux, comme défini par la marche même des promeneurs. La musique et l'auditeur sont donc en quelque sorte portés par le vent du nord, en finnois: Pohiatuuli.

Le choix du programme de ce concert d'ouverture est donc selon nous un acte symbolique. II pourrait même s'agir d'un acte critique. En effet, le monde de la musique contemporaine ne s'éloigne-t-il pas de plus en plus de l'esprit de l'avantgarde tel que défini par Pablo Picasso, Salvador Dali, Éric Satie et les autres artistes du milieu artistique parisien des années 1920 ? Les instigateurs du festival n'ont-ils pas voulu signifier que l'avant-gardisme doit rester vivant, avec sa folie et ses excès? Connaissant Walter Boudreau et sa musique, nous avons eu l'impression que c'est ce qu'il a voulu signifier à travers le «boucan » des machines du Ballet mécanique.

\section{Architectures en mouvement - VivaVoce}

L'écriture pour chœur pose de nos jours un certain nombre de problèmes au compositeur de musique contemporaine. La chorale est une institution relativement figée, comme l'est aussi l'orchestre symphonique, et la musique chorale a tendance à se mettre à la hauteur de cette institution : n'être trop difficile d'accès ni pour les choristes, qui sont souvent des amateurs, ni pour le public, souvent néophyte. Aussi, en adoptant l'écriture à quatre voix mixtes, ou même à cinq, six ou huit voix, nous faisons face à une question fort angoissante : que faire de l'harmonie? Lorsque les possibilités de timbre des instruments nous sont refusées, les fondements historiques de la musique ressortent sous leur plus simple expression et nous nous voyons presque contraints de concevoir une musique entièrement basée sur les hauteurs. C'est un rendez-vous avec l'harmonie, avec le contrepoint, avec l'histoire. 
Le programme que proposait le chœur VivaVoce présentait la démarche de trois compositeurs qui ont été confrontés à ce qui semble être de réels problèmes. Si la problématique est similaire, les réponse sont tout à fait différentes les unes des autres. André Villeneuve, avec Ecce ubi sum, choisit de s'attaquer à ce que nous considérons comme le cœur du problème en faisant du travail de l'harmonie l'une de ses préoccupations principales. Le texte chanté, de Saint-Augustin, est constitué d'une série de questions. Ces questions sont représentées en musique par des crescendos d'ensemble. L'harmonie, riche et variée, semble fortement liée aux inflexions d'intensité, donc au texte, et c'est de cette corrélation harmonie/texte, donc, dans ce cas-ci matériau/forme, que naît le discours musical, c'est-à-dire ce par quoi l'œuvre communique une signification musicale. C'est dire que la musique ne s'ajoute pas au texte mais magnifie celui-ci, accroît sa portée.

L'œuvre de Brian Cherney, Tenebrae, ajoute une dimension au problème, celle de la narration. Ici, le compositeur fait entendre certains extraits de textes qui, soit narrés soit chantés, sont écrits pour être compris. Le sens des mots étant plus univoques que celui de la musique, les mots colorent la musique et imposent une certaine attitude d'écoute à l'auditeur. L'harmonie n'est pas au premier plan, comme c'était le cas dans l'œuvre de Villeneuve, et se trouve, par le fait même, sensiblement moins diversifiée, moins changeante, moins organique en somme. Mais l'œuvre n'y perd absolument rien en poésie : l'attention est simplement mise ailleurs.

Rothko Chapel, de Morton Feldmann, s'inscrit moins directement dans la problématique traditionnelle de l'écriture chorale. La question de l'harmonie, par exemple, est résolue ici, comme dans la plupart des œuvres de Feldmann, par le concept de l'accord-objet. Le langage discursif conventionnel étant brisé, l'accord n'a plus de "fonction discursive", il est simplement un objet musical, comme un coup de cymbale ou un pizzicato d'alto. De plus, non seulement la pièce ne comporte pas de texte littéraire, mais elle ne comporte aucune référence langagière, le chœur chantant la bouche fermée tout au long de l'œuvre. Au problème de la tradition, Feldmann, comme George Antheil, donne la solution de l'avant-gardisme : il ne répond rien et fracasse simplement la question.

\section{Les voix à l'honneur - Hilliard Ensemble}

Le concert du Hilliard Ensemble offrait au public huit œuvres récentes, dont la plupart étaient franchement dans l'esprit de la musique ancienne, plus précisément de l'Ars Nova et de la Renaissance. II ne faut pas oublier que ce quatuor vocal britannique, justement spécialisé dans ce type de répertoire, emprunte son nom au joaillier et écrivain anglais Nicholas Hilliard (1547-1619). Un parfum un peu vieillot d'art british était donc au rendez-vous! Mentionnons, par exemple, les nombreuses sources 
littéraires issues des classiques anglais: The Hilliard Songbook, de Piers Hellawell, textes de Hilliard lui-même; Four Sonnets for Four Voices, de Rudolf Kelterborn, poèmes de Shakespeare; Whale Rant, d'Elisabeth Liddle, textes issus de Moby Dick; Songs of Innocence and of Experience, de José Evangelista, poèmes de William Blake. En outre, ces œuvres étaient toutes d'une facture compositionnelle proche de l'Ars Nova; pensons, par exemple, aux rythmes de gaillarde du Liddle ou aux hoquets empruntés à Guillaume de Machaut dans le Kelterborn.

Nous nous sommes demandés d'où venait cette relative uniformité esthétique. l'œuvre d'Arvo Pärt, And One of the Pharisees, était une œuvre semblable à ses autres œuvres. Cependant, un compositeur comme José Evangelista semble s'être véritablement adapté aux traits esthétiques du Hilliard Ensemble. Nous avons toujours admiré chez Evangelista le fait que sa musique, d'un style très personnel, se reconnaisse à cent lieves à la ronde; pourtant cette fois-ci, c'est plus l'indication au programme que la griffe du compositeur qui nous a indiqués qu'il s'agissait d'une œuvre d'Evangelista. En somme, disons que la couleur globale de ce concert était celle du Hilliard Ensemble et qu'il semble qu'il s'agissait davantage d'une proposition d'interprètes que d'un corpus de propositions de compositeurs. En ce sens, l'approche était diamétralement opposée à celle de l'ensemble VivaVoce.

Les enfers éternels des gens désespérés, de Paul Steenhuisen, abordait la poétique de "l'ancien" en se situant davantage au second degré. D'abord, la source littéraire : I'histoire d'Hélène, dans l'Illiade, mais racontée indirectement par Christian Bök avec la contrainte peu commode de n'utiliser que la voyelle "e". Ensuite, bien que l'utilisation de la voix ait eu un aspect archaïque, l'utilisation de sons numérisés (pour la plupart de source vocale) permettait de dérouter un tant soit peu l'auditeur. C'était, pour ainsi dire, une renaissance de la Grèce antique, mais avec les outils et les conceptions d'aujourd'hui. Une sorte de re-Renaissance.

Quant à l'œuvre d'Alexander Rastakov, Praise, elle était dans l'esprit de Pärt, mais dans une optique plus morphologique. Mentionnons l'emploi de diverses techniques vocales utilisées un peu comme des objets: trémolos très serrés, filtres avec la bouche, attaques percussives, timbres nasillards, frontaux, etc. Certains effets de masses, par exemple des accumulations en montée et raréfactions en descente, étaient très réussis. Enfin, en filigrane de ce type d'écriture, se manifestait clairement l'influence folklorique de l'Europe de l'Est. II ne faudrait pas omettre de souligner la splendide performance du Hilliard Ensemble, qui a imposé par sa pureté vocale un véritable climat de chérubins ou, du moins, d'une haute spiritualité. 


\section{Bloed / Noces de sang - McGill Contemporary Music Ensemble, Hilliard Ensemble}

C'est dans le cadre des Pays-Bas à l'honneur qu'était invité le compositeur Cornelis de Bondt, dont le festival offrait la première nord-américaine de son œuvre Bloed, précédée la veille par une causerie. L'apport de Cornelis de Bondt fut peutêtre le plus mitigé et, à ce titre, il était amusant de constater que, lors du concert, certains visages des plus illustres auditeurs étaient couverts de larmes et d'autres crispés par le dégoût. À la cigarette post-concert, les uns criaient au génie, les autres à la nullité. En réalité, il s'agissait là, en quelque sorte, d'un conflit semblable à la vieille antinomie entre positions germaniques et françaises.

Cornelis de Bondt, lors de sa causerie, avait essentiellement entretenu l'auditoire de deux thèmes : d'une part, Bloed, d'autre part, sa conception de la musique. Du développement de ces deux thèmes, nous avons personnellement retenu, en caricaturant, deux mots, respectivement "tragique» et "sale». En effet, le point de départ de Bloed est la relation du compositeur avec son père, ce dernier ayant été prisonnier dans un camp de concentration nazi. Mais il ne s'agit là que du premier degré, puisque De Bondt, ne voulant pas verser dans l'autobiographie, a cherché à hisser son propos à un niveau plus universel : celui de l'héritage humain, toujours à la croisée de la mort et de la régénération. Du reste, si l'on traduit, Bloed signifie à la fois "sang " et "génération". Voilà pour le tragique. À travers ses commentaires plus généraux et ses extrapolations, Cornelis de Bondt a aussi parlé de sa conception de la musique, que ce soit à l'égard de Bloed ou d'autres œuvres. II affirma d'abord que, pour lui, la musique est une question d'émotion, de souffle, de viscères, et qu'au moment où il écrit, il n'a aucune idée de ce qu'il fait. Bref, le pathos plus que l'ethos. En ce sens, De Bondt est très hollandais (bien que natif de Suisse), dans la mesure où il compose de manière gestuelle, à la manière d'une décharge, dans un flot presque exempt de censure. Face à cette approche, il semble impossible de ne pas faire de lien avec, en peinture, l'expressionnisme abstrait d'un Jackson Pollock ou d'un Sam Francis. Par ailleurs, De Bondt racontait avec humour qu'il était autrefois à la recherche d'une formule de la beauté, mais qu'avec le temps, il s'était plutôt mis à la recherche de l'inexactitude. Pour lui, c'est dans l'inexactitude que se déploie tout l'espace de l'expression personnelle: Bach, sous le prisme de Glenn Gould ou de Gustav Leonhardt, fait appel à deux mondes fort distincts. En somme, si le philosophe Platon était à la recherche de l'Idée parfaite, le compositeur Cornelis de Bondt est à la recherche des Ramifications inexactes. D'où, peutêtre, la part de grotesque dans son esthétique musicale. Ainsi, résumons : tragique et sale. Il y a là quelque chose qui, selon nous, aurait moins plu à Rameau qu'à Buxtehude... 
Au concert, l'œuvre était interprétée par le Hilliard Ensemble et le McGill Contemporary Music Ensemble, sous la direction de Denys Bouliane. Bloed est, en réalité, un diptyque. La première partie concerne l'héritage et consiste en un corpus d'œuvres vocales de l'Ars Nova et de la Renaissance, ponctué d'intermèdes et parfois doublé d'orchestrations de Cornelis de Bondt. II s'agit au fond d'un potpourri, dont les emprunts sont tellement crus qu'on peut d'abord être choqué. Estce une œuvre originale ou un exercice d'orchestration? Cependant, nous en sommes venus personnellement à écouter celte première partie telle qu'elle était, donc moins comme une création que comme une trajectoire dans l'histoire. De Bondt avait d'ailleurs exprimé, lors de sa causerie : "You cannot exclude the past from my ears". Ce pot-pourri était organisé en crescendo dramatique, pour mener finalement à une deuxième partie d'une extrême densité, sans citations, et construite essentiellement sur les objets ayant servi d'intermèdes et les couleurs de timbres de la première partie. Comme si le compositeur avait voulu chauffer à blanc l'inconscient collectif, pour ensuite entrer de plein fouet dans l'inconscient personnel.

Le timbre désagréable des sons échantillonnés par le synthétiseur, la couleur constamment nasillarde de l'instrumentation, l'aspect bavard de l'agogique et le peu de raffinement harmonique ont fait dire à certains qu'il ne s'agissait que d'une œuvre laide. Mais pour nous, cette œuvre était d'abord de nature expressionniste et, en ce sens, son aspect cru avait quelque chose de sublime. Les tableaux de Jérôme Bosch ne sont jamais aussi jolis que ceux de Fra Angelico, mais ils ont une violence terrible qui parfois atteint ce que nous appellerons le sublime grotesque.

\section{No man's clan - Robert Cram, Lori Freedman et leurs invités}

Au concert de Cornelis de Bondt succédait une soirée plus intimiste : celle du flûtiste Robert Cram, de la clarinettiste Lori Freedman et de leurs invités. Trois œuvres ont marqué notre attention.

D'abord, en création montréalaise, Assonance II (1989) de Michael Jarrell, pour clarinette basse, interprétée par Lori Freedman. Jarrell est reconnu comme une figure de prove de l'IRCAM; on aurait donc pu s'attendre à une musique longue, lente, flottante. Or cette œuvre, et de surcroît son interprétation, démolit ici ce préjugé réducteur et trop souvent associé à la musique "ircamienne". D'une part, la prestation de Lori Freedman avait quelque chose de Mick Jagger et, d'autre part, Assonance ll est en soi assez rock'n'roll. L'œuvre est une suite très organique de métamorphoses et d'interpolations, toujours animée par des frictions entre oppositions inhérentes (statique/dynamique, tension/détente) et constamment raffinée, au niveau du timbre, par des techniques alternatives sciemment employées lles diaphonies, notamment). 
L'œuvre de Bruce Mather Doisy Daëne (1997), pour flûte et piano est l'une de ses œuvres "vineuses", le titre correspondant au nom d'un vin de Sauternes. D'entrée de jeu, l'œuvre offre un univers harmonique à la Messiaen puis, très subtilement, franchit la porte du monde de la microtonalité. Cette transition a ceci de merveilleux qu'elle rend évident tout l'attachement que porte Mather à l'artisanat, au raffinement, à l'infiniment subtil. Mentionnons aussi ce matériau de l'œuvre, du reste assez typique chez Mather : un dialogue très rapide entre les deux instruments, canonique et très serré. Il y a là quelque chose qui sonne comme un éclat de saveur et qui nous avait d'abord fait croire que Doisy Daëne était un mousseux.

Le concert se terminant avec Attiré vers le haut par le menu (1995) de Michel Gonneville, pour alto. Cette œuvre consiste en une sorte de rondo, ayant comme refrain une petite citation de Mozart dans l'aiguë. D'autres matériaux existent, mais se font constamment avaler par ce refrain qui est répété obstinément, jusqu'à devenir franchement comique. Il s'agit d'une très belle œuvre de Gonneville, une sorte de bagatelle au discours théâtral.

\section{Montréal a rendez-vous avec Vienne - Klangforum Wien}

Yannick Plamondon et André Ristic sont deux des principaux représentants de la jeune génération de compositeurs québécois. L'un et l'autre ont reçu le Prix Jules-Léger pour la nouvelle musique de chambre, Ristic en 2000, avec Catalogue de bombes occidentales, et Plamondon en 2002, avec Autoportrait sur Times Square, la pièce qu'interprétait le Klangforum. S'il existe entre leurs musiques respectives certains liens stylistiques, du point de vue de l'attitude compositionnelle, nous avons affaire à deux mondes forts différents. La pièce de Plamondon comporte un argument politique. Le compositeur réfléchit dans son œuvre à la domination américaine sur la culture mondiale. C'est ce qui l'amène, dans un moment particulièrement savoureux de son œuvre, à citer avec beaucoup d'ironie le thème du célèbre film de science-fiction La guerre des étoiles. La Sonate de carnaval de Ristic n'est pas aussi explicite, mais nous croyons qu'elle n'est pas non plus exempte d'un certain contenu politique. Car le fait que l'œuvre fasse place à l'humour n'en fait pas pour autant une pièce légère : il se trouve dans l'œuvre des moments soudainement très introspectifs, parfois même sombres, qui sont si inattendus et semblent si "déplacés " qu'ils laissent croire à une signification cachée. Dans ce carnaval à la Ristic, il y a de tout : la fête, la joie, la folie, le doute, l'angoisse et peutêtre même la guerre, la faim, les bombes inutiles, etc. À notre avis, cette œuvre n'est pas si éloignée que son titre pourrait le laisser croire du Catalogue de bombes occidentales. Le style des deux œuvres est sensiblement le même, et les moments de "doute musical ", espaces de calme entre 
deux avalanches de sons, ces instants qui, dans le Catalogue, étaient perçus comme autant de moments d'introspection, de réflexion sur les erreurs humaines, sont toujours présents dans la Sonate de carnaval; ils sont même peutêtre plus marqués, ont plus de puissance.

L'œuvre de Plamondon est une sorte de poignard musical : elle frappe, elle remet en question notre monde en tant que monde, l'auditeur en tant qu'auditeur, elle vise à éveiller les esprits, à soulever les masses. L'arme de Plamondon contre l'impérialisme est le sarcasme, mais il s'agit malgré tout d'une attaque directe et explicite qui veut provoquer, susciter des discussions, enflammer le débat. Chez Ristic, tout est plus ambigu. Nous croyons que son "attaque» des injustices sociales passe par la mise en place du doute dans l'esprit de l'auditeur. Toutes les ambiguités formelles et harmoniques de l'œuvre visent à faire douter l'auditeur par un effet d'entraînement, car par ces ambiguitiés, la musique doute en quelque sorte d'elle-même.

Ristic et Plamondon comptent parmi les premiers compositeurs post-postmodernes du Québec. Les caractéristiques du langage musical postmoderne lemploi de citations, ironie, etc.) font également partie du langage de ces deux jeunes compositeurs mais n'y sont plus au premier plan. Les techniques et la pensée postmodernes ont été assimilées, comme les techniques et la pensée sérielles avant elles. Cela est particulièrement évident en ce qui concerne l'harmonie. Ils utilisent en effet régulièrement des harmonies classées, voir même fonctionnelles, mais cette utilisation est véritablement au service du discours et de l'expression, il ne s'agit pas d'un acte de foi comme c'était souvent le cas chez les postmodernes.

\section{Licks and Brains - Ensemble de la SMCQ, Quasar}

Le triptyque Licks and Brains, du compositeur Klas Torstensson, présenté le 7 mars par le quatuor de saxophones Quasar et l'ensemble de la SMCQ, fut sans contredit l'un des moments forts de ce festival. La musique de Torstensson est de celles dont la puissance, la vitesse et l'imprévisibilité donnent le vertige aux auditeurs. Le matériau générateur de l'œuvre est une cellule de trois sons empruntée à Arcana, d'Edgar Varèse. Cette cellule est présente dans les trois parties du triptyque et est non seulement l'élément structurant fondamental, mais aussi l'un des principaux repères perceptuels pour l'auditeur.

L'harmonie et la mélodie sont relativement secondaires dans la méthode de travail du compositeur : cette œuvre, comme la grande majorité des œuvres de Torstensson, provient d'une écriture gestuelle. C'est dire que les objets musicaux utilisés sont choisis d'abord en fonction de leurs caractéristiques énergétiques, directionnelles et de 
timbres. La méthode exposée par le compositeur pendant sa conférence du 6 mars peut se résumer en quatre étapes : 1/ conception d'un plan graphique sommaire sur une seule ligne (gestes principaux, découpage formel, etc.); 2) conception d'un plan graphique détaillé sur une seule ligne (tous les gestes, éléments d'orchestration, etc.); 3) conception d'un plan détaillé comportant une ligne par instrument lil s'agit en fait d'une partition complète mais sans hauteurs fixes : il n'y a pas de portées) ; 4) rédacfion de la partition finale. Ainsi, les hauteurs sont le dernier élément sur lequel se penche Torstensson.

Le compositeur dit de lui-même qu'il est un enfant des années 1960. C'est, à son avis, ce qui explique que, dès le début de sa carrière, il ait été animé par un sentiment de révolte face aux traditions musicales. Cette révolte s'exprime entre autres par le peu d'importance qu'il accorde aux hauteurs, faisant de celles-ci un élément secondaire, alors qu'elles étaient traditionnellement la priorité absolue des compositeurs. C'est également la très haute tension énergétique ininterrompue qui fait que ses œuvres, notamment Licks and Brains, participent à l'esprit de la révolte.

Maintenant un mot sur l'interprétation à laquelle nous avons eu droit. Quasar a rendu cette œuvre athlétique avec une virtuosité époustouflante. Tout était en place et les musiciens semblaient parfaitement à l'aise. Pour arriver à véritablement donner vie à cette œuvre qui fourmille de détails, le quatuor a dû encore une fois faire preuve d'un perfectionnisme remarquable. II serait, selon nous, temps que la carrière de Quasar débouche sur la scène internationale.

\section{$15^{\mathrm{e}}$ Concours national des jeunes compositeurs de Radio-Canada - ECM}

Pour sa $15^{\mathrm{e}}$ édition, le Concours national des jeunes compositeurs de RadioCanada offrait deux catégories : œuvres pour piano et œuvres pour ensemble. $\mathrm{Ce}$ concert présentait les finalistes de chacune des catégories.

Les trois finalistes de la catégorie d'œuvres pour piano étaient Charles-Antoine Fréchette, Matthew Rizutto et Maxime Després. L'œuvre de Fréchette (Trajectoire), interprétée par Louise-Andrée Baril, a remporté le premier prix. Elle offrait en vrac toute la palette pianistique habituelle. C'està-dire de grands brouillages lisztiens dans le grave (avec de la pédale !), des gestes pointillistes hyper/disjoints à la Boulez première manière, des effets de percussions dans le piano, des montées virtuoses, le tout en une forme d'arche très perceptible. Dans cette œuvre, Fréchette a su gérer très pertinemment son discours, avec une répartition formelle et un traitement des objets adéquats et sensible. Quant à l'œuvre Perseveration Kids de Rizutto interprétée par Brigitte Poulin, elle présente un minimalisme assez personnel. II s'agit d'une 
musique répétitive, mais dont le continuum est paradoxalement très découpé par des changements de densité et d'énergie subits. D'autre part, les matériaux ne sont pas diatoniques comme chez Steve Reich, mais plutôt éclatés et de caractère sériel. Enfin, l'œuvre de Maxime Després, interprétée par Louise Bessette, consistait essentiellement en une énorme antinomie entre le grave et l'aigu.

Passons maintenant aux œuvres pour ensemble, interprétées par l'Ensemble contemporain de Montréal. Les trois finalistes étaient Analia Lludgar, Andriy Talpash et Niklas Kambeitz. Inflexions de Lludgar, consiste effectivement en inflexions issues d'une multitude de "profilages" (d'intensités, de densités, de registres). La forme et le comportement rappelle Xenakis, l'orchestration et la couleur, Ravel. Cette œuvre a d'ailleurs remportée tous les honneurs. Puisque nous sommes dans les références, disons que l'œuvre de Kambeitz (The Days of Victory), autant par son niveau poïetique qu'esthésique, pouvait aisément faire penser à du Richard Strauss. Kambeitz s'est inspiré d'un texte du Coran et en a fait une sorte de poème symphonique très puissant, aux parfums parfois exotiques, ce qui faisait grandement penser au Zarathoustra. À travers cette œuvre, Kambeitz témoigne d'une sensibilité à fleur de peau. Quant à Talpash, il proposait avec HWY 2 une œuvre concertante où domine la clarinette basse; une œuvre bien faite, sonore et assez près des idiomes de la musique contemporaine. Enfin, mentionnons que le programme était complété par l'œuvre récipiendaire du premier prix de l'édition 1999 du concours, soit la très colorée, habile et amusante Géométrie sentimentale d'Ana Sokolovic.

\section{Triptyque intimiste - Quatuor Molinari, Trio Fibonacci, Quatuor Bozzini}

Le Triptyque intimiste était un véritable marathon de trois concerts, par trois des plus illustres ensembles de musique de chambre de la scène montréalaise : le Quatuor Molinari, le Trio Fibonacci et le Quatuor Bozzini. II s'agissait certainement d'un moment fort du festival. Nos propos se concentreront sur les compositeurs Serge Provost et Jean lesage, dont les apports au Triptyque intimiste consistaient, respectivement, en La pietra che canta et le Quatuor no. 2, deux œuvres témoignant d'un rapport différent à la tradition.

L'esthétique de Jean Lesage s'inscrit dans la mouvance des intellectuels, tel Umberto Eco, qui prétendent que la tradition ne peut être abolie et qu'elle se maintient, de manière toujours déformée, dans la mémoire présente. Le rapport au passé ne peut être qu'artificiel et, par conséquent, fait appel à un univers de masques qui, selon cette idéologie postmoderne, est de nature ludique. Ainsi, Jean lesage a développé un langage compositionnel consistant à revisiter l'histoire de la musique, mais avec une oreille contemporaine. D'où les évocations stylistiques, les mosaïques d'allusions. Ainsi, par 
exemple, le dernier mouvement de son Quatuor no. 2 consiste en un faux menuet de forme rondo qui s'intitule "Musique sur laquelle il n'est pas interdit de danser ». En fait, on pourrait dire, en paraphrasant lesage, que sa musique est un théâtre aux personnages multiples et contradictoires, dont la scène est la mémoire collective. Notons qu'il était très intéressant d'entendre, dans la même soirée, le trio avec piano Fremde Szenen, de Wolfgang Rhim, dont l'esthétique est proche de celle de lesage. Cette œuvre est pleine d'une schizophrénie à travers laquelle on a l'impression d'écouter du Schumann avec la folie de Schumann.

Chez Serge Provost, le rapport à la tradition est tout aussi présent, mais de nature très différente. Alors que Lesage sort carrément les morts de leurs tombes et les fait danser entre eux, Provost a plutôt tendance à laisser les morts parmi les morts; en revanche, il humera volontiers le doux parfum des dernières héritières des défunts, à savoir les fleurs. En d'autres termes, lesage donne les morts en spectacle alors que Provost s'en imprègne. Ainsi, dans la Pietra che canta, Provost a utilisé l'électronique et le traitement en temps réel pour évoquer l'émotion que lui avait fait vivre sa découverte, tout à fait par hasard, de la tombe de Claudio Monterverdi, dans une église de Venise à l'acoustique particulière. De Venise, un lien s'est créé dans l'imaginaire de Provost avec Luigi Nono et ses musiques organisées, formellement, en îlots, comme autant de métaphores urbanistes de la configuration de cette ville serpentée de canaux. Toujours dans l'imaginaire "provostien", s'est créé un autre lien avec Venise : une cellule issue de Richard Wagner - Venise, de Franz liszt. Puis encore un lien, cette fois entre Liszt et Venise : Pétrarque, le grand poète vénitien. Et encore un lien, entre Liszt et Pétrarque, avec l'évocation d'un Sonnet de Pétrarque signé Liszt. Et ainsi de suite, jusqu'à ce que le compositeur retire de ce circuit imaginaire non seulement une poétique, mais aussi une pensée formelle et un bon corpus de matériaux pré-compositionnels. En somme, le rapport à la tradition consiste en une errance libre, intime et personnelle dans la culture, la mémoire, voire l'imaginaire, et ne cherche pas à être nécessairement perceptible pour l'auditeur. Au même titre que pour lesage et Rhim, il était intéressant d'entendre, dans la même soirée, la Pietra che canta, de Serge Provost, et le quatuor à cordes Fragmente - Stille, An Diotima, de Luigi Nono.

\section{Corps et âmes - OSM}

Michel Longtin et Sean Ferguson ont une préoccupation commune : la science. Cependant, leur approche de la science, leur attitude face à elle, est radicalement opposée. Ainsi, Longtin aborde la science par l'intermédiaire du symbolisme musical, du mysticisme en quelque sorte, tandis que Ferguson a une approche très rigoureuse, une véritable approche scientifique.

L'œuvre In the Flesh, de Sean Ferguson, comme pratiquement toute sa musique, est basée sur des recherches approfondies sur les modèles psychoacoustiques, par- 
ticulièrement en ce qui concerne l'harmonie. Ces recherches visent à établir une véritable syntaxe harmonique qui, comme la syntaxe tonale, gère efficacement les tensions et les résolutions harmoniques. Ferguson cherche à réunir de façon cohérente et personnelle le plaisir cognitif et le plaisir physique (ou sensuell que procure la musique. C'est précisément pour mieux comprendre ce plaisir physique, pour connaître ses modes d'apparitions et sa provenance que le compositeur s'intéresse à la psychoacoustique. Le principal enjeu de In the Flesh est de nature téléologique : il s'agit d'un parcours vers le climax final. Ce parcours n'est pas unidirectionnel, il est fait de quatre courbes croissantes d'intensité dont la dernière aboutit au climax, les trois premières étant des sortes de feintes. Ferguson joue donc avec les attentes et les réflexes des auditeurs. En effet, les trois feintes successives, ces climax non aboutis, font en sorte que le climax final est d'une intensité nettement plus forte, qu'il est plus efficace.

L'œuvre de Michel Longtin se situe dans un tout autre registre. Le compositeur nous propose un parcours autobiographique à travers les gens, les œuvres et les moments qui ont marqué sa vie. Quarternions est donc d'abord et avant tout une œuvre symboliste à l'intérieur de laquelle sont représentés non seulement des équations chimiques et mathématiques, mais aussi des situations et des récits poétiques. Le mode et la forme de ces représentations sont souvent naifs et toujours charmants, tous empreints de l'atmosphère poétique qu'ont en commun toutes les œuvres de Longtin et que son grand talent d'orchestrateur met si bien en évidence.

\section{En guise de conclusion}

MNM se voulait au départ une fenêtre ouverte sur la nouvelle musique québécoise et canadienne, une sorte de panorama des différentes tendances de la création musicale. Nous constatons que les organisateurs du festival ont choisi, à tort ou à raison, de ne pas inclure dans la programmation les œuvres de compositeurs tels que Gilles Tremblay, Serge Garant et François Morel, compositeurs qui sont en quelque sorte les initiateurs de la période de "maturité " de la musique contemporaine québécoise. Les "aînés" sont donc pratiquement absents de la programmation. On a cependant fait une bonne place aux "cadets", notamment à André Ristic, à Yannick Plamondon et aux finalistes du concours des jeunes compositeurs de RadioCanada. Si ce panorama n'est pas tout à fait complet let on peut à juste titre se demander s'il aurait été possible qu'il le soit) il a le grand mérite d'avoir peu tenu compte des partis-pris esthétiques, présentant un état des lieux autant de la musique contemporaine que de la musique actuelle, de l'électroacoustique et de la musique techno. II semble donc y avoir eu un véritable effort pour que la programmation du festival soit en accord avec son mandat. 
En tant qu'auditeur, nous ne pouvons parler de ce festival que comme d'une réussite. La programmation dans son ensemble était contrastée et équilibrée. Les interprétations étaient pratiquement toutes de très haut niveau. Les concerts de MNM ont sans aucun doute été à l'origine de plusieurs chocs, révélations, illuminations, etc. II reste maintenant à savoir si le désir plus ou moins caché des organisateurs du festival, à savoir que $M N M$ devienne une véritable tradition, deviendra réalité. Nous avons la conviction qu'à l'issue de cette première édition, tout le milieu montréalais de la musique contemporaine est prêt à mettre de nouveau la main à la pâte pour le prochain festival MNM. 

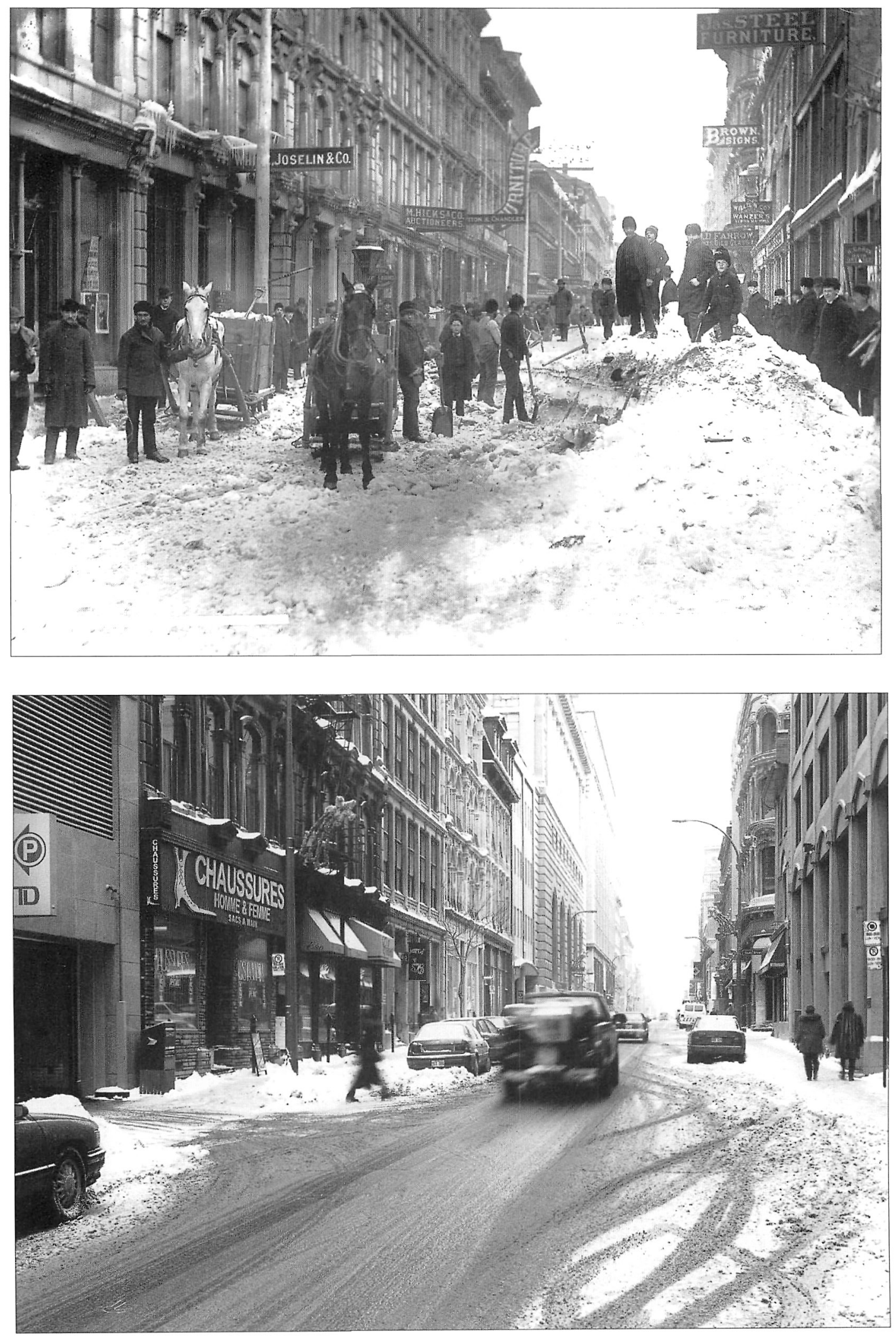